\title{
Demographic Variables and Self-Management Practices as Correlates of Glycemic Control among Reproductive Age Diabetic Women in a Tertiary Hospital in Ile-Ife, Osun State, Nigeria
}

\author{
Christiana Olanrewaju Sowunmi ${ }^{1}$, Oluwatoyin Abiola Lanre-Ogungbile ${ }^{2}$, Christiana Adetoun \\ Owopetu $^{3}$, Jacob Kehinde Opele ${ }^{4 *}$ \\ ${ }^{1,2,3}$ School of Nursing, Babcock University, Ilishan-Remo, Ogun State, Nigeria \\ ${ }^{4}$ Department of Library and Information Science, Federal University, Oye-Ekiti, Nigeria
}

\begin{abstract}
Glycemic control remains a major cornerstone in the management of diabetes mellitus. Despite consistent studies, the incidence of both short-and long-term complications of diabetes had been quite alarming. This study employed a descriptive design. A total enumeration of 185 diabetic women of reproductive age constituted the sample size. A self-structured questionnaire with 40 items divided into five sections and a checklist for recording glycemic control were used for data collection. The data analysis involves the use of Statistical Package for Social Sciences (SPSS) version 23. Results were presented as frequencies, mean and standard deviation, and regression analysis. The findings from the study revealed that 112 (67.1\%) of the women had normal glycemic status while 55 (32.9\%) had high status. Furthermore, it revealed a significant negative correlation between educational qualification and glycemic control ( $r=-.161, p=0.049)$. The study revealed a slight significant correlation between diet and glycemic control ( $r=-.148, p=0.052)$. Other practices did not share a significant correlation with glycemic control. The study concluded that an appreciable percentage of the respondents had high glycemic control status, education and diet had a significant partial correlation with glycemic control. Most of the women had good practice of diet, exercise, medication, and self-monitoring of blood glucose as shown in their health records. However, a sharp contrast exists between their record of glycemic control, which was good, and the current level, which revealed no significant correlations between selfmanagement practices and glycemic control. Hence, the need for more patient education on selfmanagement practices.
\end{abstract}

Keywords: Diabetic women, glycemic control, Reproductive age, Self-Management practices, Tertiary hospital.

\section{Introduction}

Diabetes is a rapidly emerging condition across the globe that has been an issue of concern because of its negative effect on the individual, the family, the health system, and the society as a whole known to a layman as 'sugar diabetes', its occurrence is progressively high and common among individuals of different age groups. These issues create a burden on a patient with diabetes mellitus and, if not properly handled, could result in loss of interest about one's health or frustrations with the end product of deteriorating health. The above showed the need to periodically checkmate this issue associated with managing diabetes. Glycemic control remains the cornerstone of diabetes care which requires balancing insulin replacement with diet, medication, and exercise. This goal of maintaining a normal glucose level requires education, decision strategies, self-care or monitoring, and ultimate avoid hyper and hypoglycemia.

*Corresponding Author: jacob.opele@fuoye.edu.ng 
Glucose level has to be continuously controlled and without holiday because failure to do so may result in biological and psychosocial problems. Glycemic control is the central focus of treatment among patients with diabetes mellitus and prevents other induced conditions resulting from diabetes mellitus. It is a hallmark of regulating typically the blood sugar (glucose) in a person with diabetes mellitus to maintain a normal glucose level $(70-130 \mathrm{mg} / \mathrm{dl}$ or 3.9 $7.2 \mathrm{mmol} / \mathrm{L}$ ) [1]. However, the need for glycemic control is imperative as it helps in a managerial condition where the body's ability to process blood glucose is impaired due to both lifestyles and health problems [2]. Thus, the act of controlling blood glucose levels will help in reducing diabetic complications which is a rapidly emerging condition across the globe [3].

Globally in 2016, 1.6 million deaths were attributed to diabetes. Similarly, in 2015, the report showed that 40 million deaths resulted from non-communicable diseases, which corresponded to $70 \%$ of all deaths worldwide [3]. Furthermore, the World Health Organization estimates that more than 346 million people worldwide have DM [3], and this number is likely to be more than double by 2030 without any intervention. Thus, diabetes among the reproductive age group calls for more attention due to high morbidity and mortality rate within this age group and needs to assess the selfmanagement practices of women of reproductive age.

The needs of diabetic patients are not only limited to adequate glycemic control but also correspond with preventing complications, disability limitation, and rehabilitation. DM is a lifelong condition and leads to chronic complications if blood glucose is constantly elevated [3]. Diabetes can influence everyday social interactions in many ways; patients experience food intake restrictions, consistency in times for monitoring blood glucose levels and adherence to medication regimen all interfering with patients' social activities [2]. This study was limited to two glycemic control methods: fasting plasma glucose test and 2 Hours Post Prandial Random plasma test due to its affordability strength among respondents. A fasting plasma glucose test is done at least eight hours after the last meal. According to the World Health Organization, fasting plasma glucose level $7.0 \mathrm{mmol} / \mathrm{l}$ or more meets the criteria for the diagnosis of diabetes [3].

\section{Statement of the Problem}

There is an increased prevalence rate of diabetes mellitus (DM) with associated poorly managed status [4]. DM is the sixth leading cause of death worldwide and one of the most prevalent chronic diseases affecting about 425 million adults worldwide [3]. In Africa, out of 425 million affected by DM worldwide, more than 16 million with a prevalence rate of $3.9 \%$ and 3,21,000 deaths were attributed to diabetes. In a study conducted at Ogun State, Southwest Nigeria as their population asserts that female preponderance for diabetes with the majority (63.4\%) having no formal education, $8.9 \%$ earning less than NGN 18,000 monthly income, putting diabetes prevalence rate at $4.6 \%$. Similarly, a $3.7 \%$ prevalence rate of diabetes concerning age and gender was reported in Osun State, Southwest Nigeria, noting diabetes as predictive to chronic kidney disease [5, 6, 7]. Also, The America Diabetic Association asserts that $70-110 \mathrm{mg} / \mathrm{dl}$ equivalent of $4-6 \mathrm{mmol} / 1$ sugar level meet criteria for diagnosing fasting blood sugar. Besides, it has been proven that two hours postprandial random plasma test is the simplest test and does not require fasting before taking the test. America Diabetic Association value of $180 \mathrm{mg} / \mathrm{dl}(10 \mathrm{mmol})$ or if $200(11.1 \mathrm{mmol} / \mathrm{l})$ or more than $200 \mathrm{mg} / \mathrm{dl}$ of blood glucose indicates diabetes but has to be reconfirmed [8].

The burden of DM is attributable to complications (acute or chronic). Prolonged uncontrolled high blood glucose levels damaging the heart, blood vessels, eyes, kidneys, and nerves leading to disability and premature death (016). The disruption results from the physical limitations imposed on the affected 
individuals by the disease, as well as from the social and cultural implications of living with diabetes such as loss of sexual function, blindness, and loss of limb resulting in loss of sense of identity in individuals and change in social relationships [9]. Participants in the Diabetes Attitude Wishes and Needs (DAWN) Study reported anxiety, fear, and worry about diabetes complications, depression, and a sense of hopelessness as challenges; they also reported being discriminated against in the workplace [10].

Diabetes has no cure but is managed throughout the life of affected individuals with medications and lifestyle modification measures to maintain reasonable glycemic control, reduce the risk of complications, and improve the quality of life [3]. The incidence of diabetes cut across different age groups. In 2015, adults aged 45-64 were the most diagnosed age group for diabetes; new cases of DM from age 18 years distributed as 18-44(355,000), 45-64 (809,000), and 65-above $(366,000)$ [8].

In the face of scarce resources and a weak healthcare system, the burden of diabetes mellitus has left a mark amongst reproductiveage women, and its impact is far-reaching as it affecting their immediate family; adding to financial burden considering the massive amount of their income spend on medication, psychological implication as well as a reduction in women quality of life [11]. Furthermore, many adverse outcomes are associated with poor diabetes control and especially among reproductive-age women concerning fertility, pregnancy, and labour. Many women with preexisting diabetes mellitus are not prepared for pregnancy before conception, a situation that results from unintended pregnancies, leading to infant morbidity and mortality and several congenital disabilities, thus putting women of reproductive age at a higher risk of losses and reduced life expectancy [12].

\section{Research Question}

What is the percentage of diabetic women of reproductive age with normal glycemic control?

\section{Hypotheses}

Ho1: There is no significant relationship between socio-demographics and glycemic control among diabetic females of reproductive age.

Ho2: There is no significant relationship between individual self-management practices and Glycemic Control among diabetic females of reproductive age.

Ho3: There is no significant influence of combined self-management practices on glycemic control among diabetic females of reproductive age.

\section{Materials and Methods}

This study employed a descriptive design to assess self-management practices as correlates of glycemic control amongst diabetic women of reproductive age in a selected tertiary hospital in Osun State. The total enumeration method was used to select 167 respondents obtained from a five- week record of clinic attendance in endocrinology unit of the selected hospital. In line with adhering to the ethical guidelines that guide the conduct of research, this study sought permission for ethical clearance from the Babcock University Research Ethical Committee (BUHREC). Health research ethics committee of the study centres was also approached through an application letter from the School of Nursing, Babcock University Ilishan-Remo, Ogun State.

Permission letter was received by the health research ethics committee of the study centres and was taken to the Head of Department, Internal Medicine, Endocrinology Unit. Consent of all participants was obtained before the conduct of the research. The researcher pledged to keep the entire information gathered from 
them in confidence. The study involved the use of quantitative data only. The quantitative data was obtained through the use of a self-structured questionnaire which consisted of five sections for primary data based on socio-demographics, self-management practices, and checklist for secondary data on information that pertains to the clinical history of respondent's glycemic level. The researcher examined the entire instrument for completeness, and those not properly filled were excluded. Out of 185 questionnaires given out, the researcher was able to retrieve 167 , which were coded and analyzed using the computer social package statistical software (SPSS Version 23). Descriptive statistics of percentages, mean and standard deviation were used to answer the four research questions, while correlation and multiple regression statistics were used for testing the hypotheses guiding this study at a significance level of 0.05 .

Table 1. The Glycemic Control among Diabetic Women of Reproductive Age in OAUTHC

\begin{tabular}{|l|l|l|}
\hline Glycemic status & Frequency & Percentage \\
\hline Low $(<4.5)$ & 0 & 0.0 \\
\hline Normal $(4.51-10.3)$ & 112 & 67.1 \\
\hline High $(>10.3)$ & 55 & 32.9 \\
\hline Total & $\mathbf{1 6 7}$ & $\mathbf{1 0 0 . 0}$ \\
\hline
\end{tabular}

\section{Results}

\section{Analysis of Research questions and hypotheses}

Research Question 1: What is the percentage of diabetic women of reproductive age in OAUTHC with normal glycemic control?
In order to answer this research question, a descriptive statistical analysis was carried out to generate frequency and percentage. Table 1 revealed that the majority $(67.1 \%)$ of the women had normal glycemic status while $32.9 \%$ had high status and none had low status. This implies that most of the women followed their medical advice about self-management practices of glycemic control which led to normal practice.

Table 2. Correlation Between Socio-Demographics (Age, Education, Income, Parity, etc.) and Glycemic Control among Diabetic Females of Reproductive Age

\begin{tabular}{|l|l|l|l|l|l|}
\hline Correlation & Mean & Std. Dev & r & p value & Remarks \\
\hline Age and glycemic control & 3.5269 & 1.17101 & -.133 & .086 & Not sig. \\
\hline $\begin{array}{l}\text { Educational Qualification and } \\
\text { glycemic control }\end{array}$ & 3.1796 & 1.07157 & -.161 & $.049^{*}$ & sig. \\
\hline $\begin{array}{l}\text { Average Monthly Income and } \\
\text { glycemic control }\end{array}$ & 1.8323 & 1.07352 & .049 & .532 & Not sig. \\
\hline Parity and glycemic control & 2.1138 & 1.25352 & -.130 & .095 & Not sig. \\
\hline
\end{tabular}

*Correlation is significant at the 0.05 level (2-tailed).

\section{Testing of Hypotheses}

The three hypotheses were tested at 0.05 level of significance.

Ho1: There is no significant relationship between socio-demographics and glycemic control among diabetic females of reproductive age in the study setting.
The hypothesis was tested using correlation analysis. Table 2 revealed a negative nonsignificant correlation between Age $(r=-0.133$, $\mathrm{p}>.05)$; parity ( $\mathrm{r}=-0.130, \mathrm{P}>.05)$ and glycemic control. Furthermore, it revealed a positive nonsignificant correlation between average monthly income and glycemic control $(r=0.049, \mathrm{p}<.05)$ 
among women of reproductive age at the Obafemi Awolowo University Teaching Hospitals Complex (OAUTHC), Ile-Ife. However, it is interesting to note that the study revealed a significant negative correlation between educational qualification and glycemic control ( $\mathrm{r}=-.161, \mathrm{p}<.05)$.

Table 3. The Pearson Correlation between Individual Self-Management Practices and Glycemic Control

\begin{tabular}{|l|l|l|l|l|l|}
\hline Self-management practices & Mean & Std. Dev & R & P-value & Remarks \\
\hline Diet and Glycemic Control & 25.2395 & 1.24769 & .148 & .052 & sig. \\
\hline Exercise and Glycemic Control & 30.7844 & 6.14398 & -137 & .076 & Not sig. \\
\hline Medication and Glycemic Control & 21.2874 & 5.33470 & -.065 & .407 & Not sig. \\
\hline $\begin{array}{l}\text { Self-monitoring of blood glucose } \\
\text { and Glycemic Control }\end{array}$ & 17.1257 & 1.46928 & -.092 & .239 & Not sig. \\
\hline
\end{tabular}

*Correlation is significant at the 0.05 level (2-tailed)

The hypothesis was tested using correlation analysis. Table 3 revealed a significant positive correlation between diet and glycemic control ( $\mathrm{r}$ $=.148, \mathrm{p}<.05)$, while it reveals non-significant negative correlation between exercise $(\mathrm{r}=$ 0.137, $\mathrm{p}>0.05)$; medication $(\mathrm{r}=-0.065, \mathrm{p}>0.05)$; self-monitoring of blood glucose $(r=-.092$, $\mathrm{p}>.05)$ and glycemic control among women of
Ho2: There is no significant relationship between individual self-management practices and glycemic control among diabetic females of reproductive age

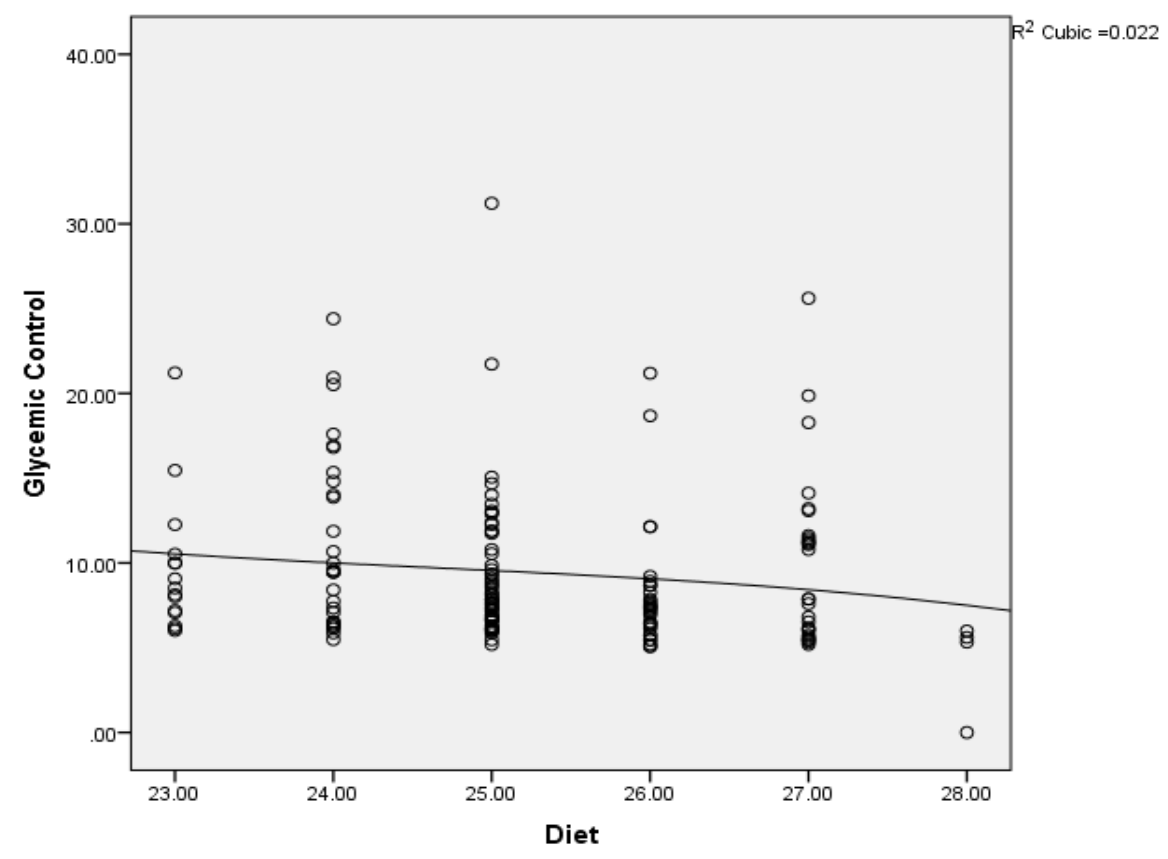

Figure 1. Correlation Between Diet and Fasting Blood Sugar among Diabetic Women of Reproductive Age 


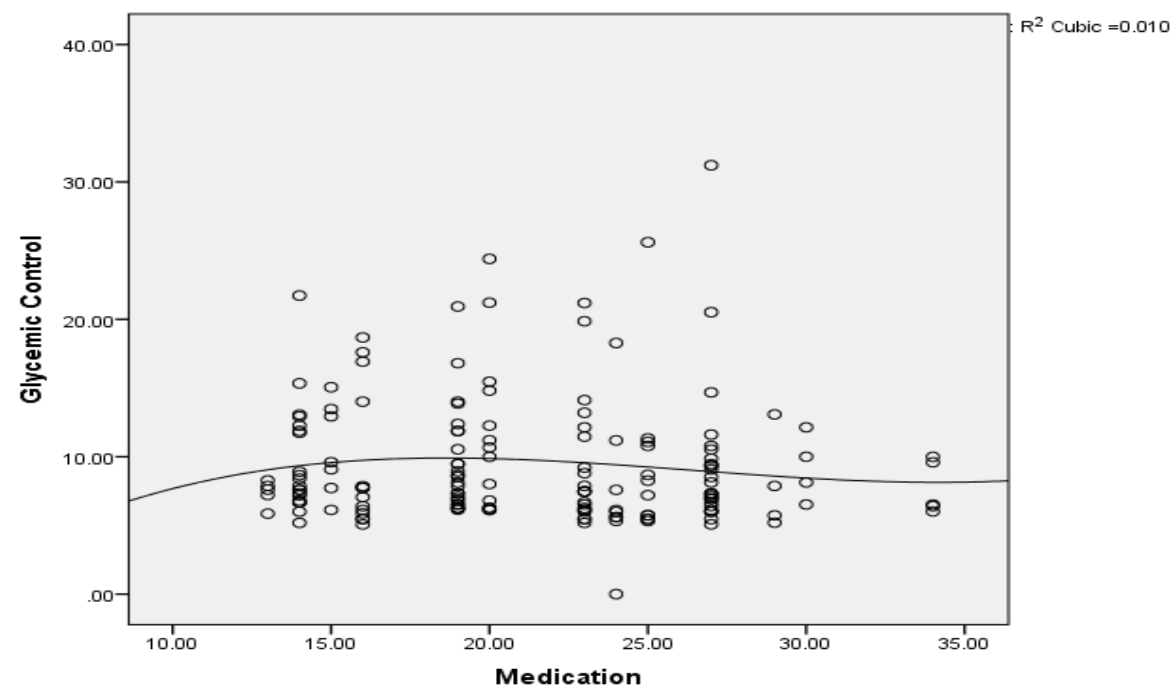

Figure 2. Correlation between Medication and Glycemic Control among Diabetic Females of Reproductive Age

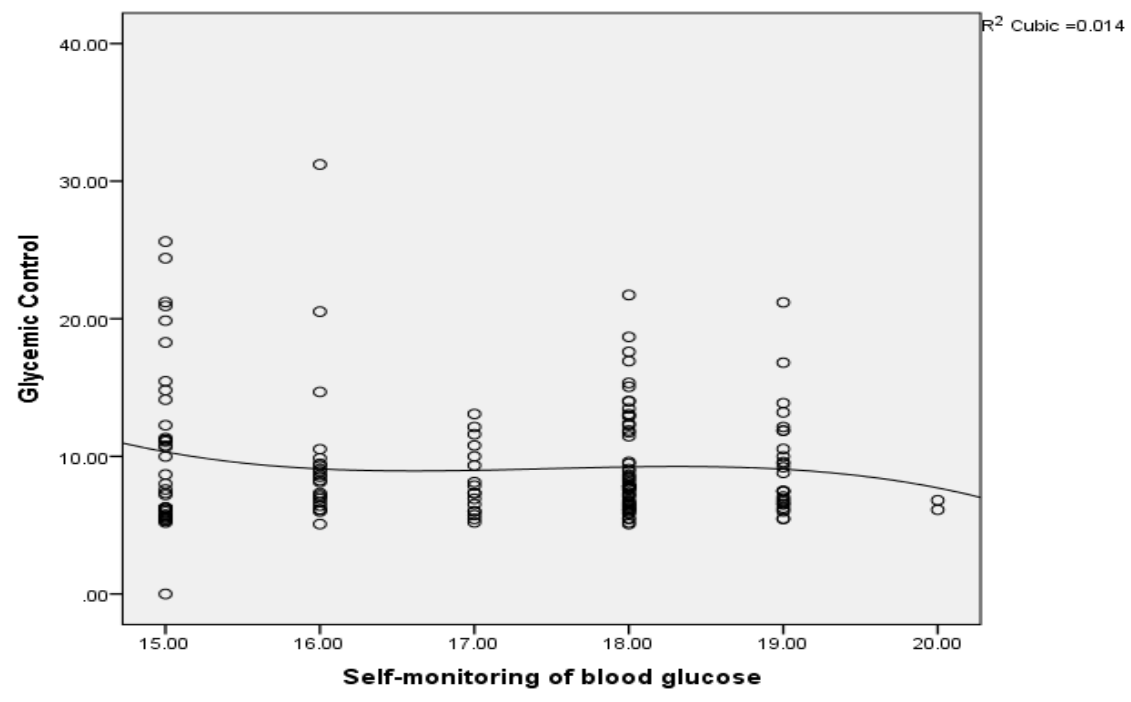

Figure 3. Correlation between Self-Monitor of Blood Glucose and Glycemic Control

Ho3: There is no significant influence of combined self-management practices on glycemic control among diabetic females of reproductive age

Table 4. Regression Analysis of the Influence of Combined Self-Management Practices on Glycemic Control among Diabetic Females of Reproductive Age at OAUTHC

\begin{tabular}{|l|l|l|l|l|l|}
\hline \multirow{2}{*}{ Model } & Unstandardized Coefficients & Standardized Coefficients & t & Sig. \\
\cline { 2 - 7 } & B & Std. Error & Beta & & 3.515 \\
\hline (Constant) & 31.964 & 9.094 & & -001 \\
\hline Diet & .502 & .290 & -.138 & -1.731 & .035 \\
\hline Exercise & -.105 & .089 & -.142 & .186 & .237 \\
\hline Medication & .015 & .104 & .018 & -1.607 & .110 \\
\hline $\begin{array}{l}\text { Self-monitoring of } \\
\text { blood glucose }\end{array}$ & -.408 & .254 & -.132 & & \\
\hline
\end{tabular}




$\begin{array}{lll}\mathrm{R} & = & 0.226 \\ \mathrm{R}^{2} & = & 0.051 \\ \text { Adjusted } \mathrm{R}^{2} & = & 0.027 \\ \mathrm{~F}(4,162) & = & 2.172 \\ \mathrm{p} \text {-value } & = & 0.074\end{array}$

The hypothesis was tested using regression analysis. Table 4 revealed that the four diabetic self-management practices explained $5.1 \%$ of the variance explained in the glycemic control. It revealed that the f-statistics; $F(4,162)=2.172$ with its corresponding probability of 0.074 . This means that there is an overall weak relationship between diet, exercise, medication, selfmonitoring of blood glucose, and glycemic control. In addition, the table revealed that only $\operatorname{diet}(\beta=-502, \mathrm{t}=-1.731, \mathrm{p}<.05)$ significantly correlated with glycemic control among diabetic women of reproductive age. Others such as exercise $(\beta=-.105, \mathrm{t}=-1.186, \mathrm{p}>.05)$; medication $(\beta=.105, \mathrm{t}=.145, \mathrm{p}>.05)$ and selfmonitoring of blood glucose $(\beta=-.408, t=-$ 1.607, $\mathrm{p}>.05)$ did not significantly influence glycemic control. Implying that glycemic control of most of the patients is based on diet. That is, they tried hard to follow their doctors' advice on the choice of diets most appropriate for them.

\section{Discussion}

Hypothesis one revealed a negative nonsignificant correlation between Age; parity, and glycemic control. The findings also revealed a positive non-significant correlation between average monthly income and glycemic control among women of reproductive age. On the contrary, it revealed a significant negative correlation between educational qualification and glycemic control ( $\mathrm{r}=-.161, \mathrm{P}<.05)$, implying that educational qualification among diabetic females of reproductive age at OAUTHC was a major predictor of glycemic control. This means that education plays a significant role in adherence to medical advice among diabetes patients in the study area. In a related study, [4] the importance of patient education for better outcomes of self- management of diabetes was documented. That is, patient education is an integral component of high-quality diabetic care. Besides, it can be said that diabetes education programme may help patients to have a practical understanding of approaches to self-management of diabetes and related conditions. Knowledge and understanding are derived from education and are important elements in moving an activated patient towards better self-management of diabetes.

Hypothesis 2 revealed a significant positive correlation between diet and glycemic control; it also reveals a non-significant negative correlation between exercise, medication, selfmonitoring of blood glucose and glycemic control among women of reproductive age at the Obafemi Awolowo University Teaching Hospitals Complex. On the contrary, the study revealed a significant correlation only between diet and glycemic control among women of reproductive age at the Obafemi Awolowo University Teaching Hospitals Complex. Again, a good diet is seen as a major remedy for improving self-care management practices. The last hypothesis revealed that the four diabetic self-management practices explained only $5.1 \%$ of the variance in glycemic control. In other words, the current study shows that there is a weak relationship between diet, exercise, medication, self-monitoring of blood glucose, and glycemic control. It is worrisome that out of the four diabetic self-management practices, the only diet was shown to significantly influence glycemic control among diabetic women of reproductive age at OAUTHC.

The overall outcome of this research shows that self-management of DM remains the cornerstone of diabetes care. Studies have shown that self-care behaviours that patients with T2DM must learn or modify include eating healthily, undertaking physical activities, following a prescribed medication regimen, and self-monitoring their blood glucose level [13]. Therefore, every effort should be made by all involved with diabetes care to support self- 
monitoring blood glucose (SMBG) as part of an overall self-management strategy. Further, literature has also shown that self-monitoring of blood glucose and regular blood pressure (BP) measurement is essential for the better wellbeing of DM [5].

Self-monitoring blood glucose has proven effective for patients with type $1 \mathrm{DM}$ as well as patients with type $2 \mathrm{DM}$. The patient might cope more independently with their disease with selfmonitoring of blood glucose. They might achieve a better understanding of the factors that affect their disease and potentially a betterperceived quality of life. Therefore, when people keep records of their behaviours in the form of a diary or checkmarks on their calendar, they become aware of gains and deficits, which leads them to take further action. In the study cited by Ralf, Agata, \& Maryam, 2014, an active control intervention (self-monitoring tool for dental flossing) was conducted; self-monitoring had a beneficial effect only for those students who were already somewhat motivated to increase their oral self-care. In other words, selfmonitoring worked in the volition (postintentional) stage of the participants but not in the earlier motivation (pre-intentional) stage.

\section{Summary of Findings}

It has been established in the current study that the need for good glycemic control is imperative as it helps in the managerial condition where the body's ability to process blood glucose is impaired due to both lifestyles and health problems [3]. Thus, the act of controlling blood glucose levels will help in reducing diabetes which is a rapidly emerging condition across the globe. Besides, studies have shown that self-care behaviours among patients with T2DM must include eating healthily, undertaking physical activities, following a prescribed medication regimen, and selfmonitoring their blood glucose level [13]. The findings from the current study revealed that about two-thirds majority of the women had normal glycemic control based on their past clinical history (medical records). Besides, the study revealed a significant correlation between diet and glycemic control among diabetic females of reproductive age.

On the other hand, it indicated that exercise, medication, and self-monitoring practices though at a moderate level among the patients, did not have a significant influence. The study shows no significant correlation between parity and glycemic control among diabetic females of reproductive age in a selected hospital in Osun State. Lastly, the study revealed that although most of the patients had good diabetes selfmanagement practices, however, there seems to be a disparity between their past medical records history of diabetes status and the outcome of their self-management practices based on their responses to the questionnaires administered. That is, their medical records indicated normal glycemic control while the outcome of the questionnaire analysis revealed moderate practices of diet, exercise, medication, and selfmonitoring of blood glucose level.

\section{Conclusion}

The findings from the current study revealed that diet, exercise, medication, and selfmonitoring of blood glucose are essential for glycemic control among diabetic women of reproductive age at the Obafemi Awolowo University Teaching Hospitals Complex. The findings from the study revealed that about twothirds majority of the women had normal glycemic control based on their past clinical history (medical records). Also, the study revealed a significant correlation between diet and glycemic control among diabetic females of reproductive age. On the other hand, it indicated that exercise, medication, and self-monitoring practices though at a moderate level among the patients, did not have a significant influence. The study shows no significant correlation between parity and glycemic control among diabetic females of reproductive age in a selected hospital in Osun State. Lastly, the study revealed that although most of the patients had 
good diabetes self-management practices, however, there seems to be a disparity between their past medical records history of diabetes status and the outcome of their self-management practices based on their responses to the questionnaires administered. That is, their medical records indicated normal glycemic control, while the outcome of the questionnaire analysis revealed moderate practices of diet, exercise, medication, and self-monitoring of blood glucose level.

\section{Acknowledgements}

The authors wish to thank all institutions and respondents that allowed us to collect their

\section{References}

[1] Witkowski A, et al. (2011). Effects of renal sympathetic denervation on blood pressure, sleep apnea course, and glycemic control in patients with resistant hypertension and sleep apnea. Hypertension; 58:559-65.

[2] Al-Goblan, A. S., Al-Alfi, M. A., \& Khan, M. Z. (2014). Mechanism linking diabetes mellitus and obesity. Diabetes Metab Syndrome.

[3] World Health Organization (WHO) (2018). World health statistics 2017: Monitoring health World Health Day (2016). Beat Diabetes. Global Report on Diabetes. License: CC BY-NC-SA 3.0 IGO.https://www.who.int/about/whoweare/publishing-policies/copyright.

[4] Ayesha, O. (2015). Reproductive knowledge and use of contraception among women with diabetes. South African Med J .9.

[5] Kisokanth, G., Prathapan, S., Indrakumar, J., \& Joseph, (2013). "Factors influencing selfmanagement of diabetes mellitus; a review article" journal of diabetology 3, (1). Vol-3 Pg. 6.

[6] Rasaki O. S, Kasali, F. O, Biliaminu, S. A, Louis, O. O, Abdullateef, G. S. \& Yusuf, M. (2017). Prevalence of diabetes and pre-diabetes in Oke-Ogun region of Oyo State, Nigeria. Journal of medical research and Health Edu.

[7] Oluyombo, R., Ayodele, P. O., Akinwusi, O. O., Okunola, A., Arogundade, F. A., Sanusi, A. A. \& personal health information for prompting the conduct of this study.

\section{Disclosure}

The authors have declared no conflicts of interest for this article.

\section{Author Contributions}

All the authors have contributed to this study in ways that conform to Texila authorship criteria. All authors had input in article draft/ revision and approved the final version of the manuscript.

Onayade A. (2013). A Community Study of the Prevalence, Risk Factors, and Pattern of chronic kidney disease in Osun State, southwest Nigeria. West African journal of medicine.2013;32(2):85-92. [8] Center for Disease Control and Prevention, National Diabetes Statistics Report, 2014 \& 2015 (US Department of Health and Human Services, Center for Disease Control and Prevention, Atlanta, 2014). [9] De-Graft, A. A., Owusu-Dabo, E., Agyemang, C. (2013) Diabetes in Ghana: a review of research on prevalence, experiences, and healthcare. Available from https://www.researchgate.net/

[10] Stuckey, H. L., Mullan-Jensen, C. B., \& Reach, G. (2014) personal accounts of the negative and adaptive psychosocial experiences of people with diabetes in the second Diabetes Attitudes, Wishes and Needs (DAWN2) study. Diabetes Care; 37, 2466-74. type 2 diabetes mellitus: present and future perspectives. Nature reviews.

[11]Gurmu, Y., Gela, \& Aga, (2018) Factors associated with self-care practice among adult diabetes patients in West Shoa Zone, Oromia Regional State, Ethiopia BMC Health Serv Res. 18, 732 (2018). https://doi.org/10.1186/s12913-018$3448-4$.

[12] Tennant, P. W., Bilous, R. W., Prathapan, S., \& Bell, R. (2105). Risk and recurrence of serious adverse outcomes in the first and second pregnancies 
of women with preexisting diabetes." Diabetes Care. 38, 610-619.

[13] Yekta, Z., Pourali, R., Aghassi, M. R., Ashragh, Ravanyar, L., \& Rahim, M. Y. (2015). “Assessment of self-care practice and its associated factors among diabetic patients in Urban are of Urmia, Northwest of Iran" Journal of Research in Health Science 11, 1-4. 